\title{
The Effect of Partial Substitution of Sulfur on the Structural Properties of Amorphous Selenium
}

\author{
Ali Abou Shama \\ Physics Department, Faculty of Science, Ain Shams University, \\ 11566 Abbassia, Cairo, Egypt.
}

\begin{abstract}
Amorphous samples of $\mathrm{Se}, \mathrm{SSe}_{40}, \mathrm{SSe}_{30}$ and $\mathrm{SSe}_{10}$ were prepared as bulk glasses by air quenching. The structural properties of these samples were studied applying X-ray diffraction using $C u$ - $k_{\alpha}$ radiation. The $R D F$ analysis of the short range order (SRO), revealed no significant change in the bond length of the Se-Se pairs upon S substitution. The second nearest neighbor suffered a systematic increase in the bond length, where it increases from $3.697 \AA$ for pure Se to 3.896, 3.865 and $3.853 \AA$ for $\mathrm{SSe}_{40}, \mathrm{SSe}_{30}$ and $\mathrm{SSe}_{10}$, respectively. A similar change in the third nearest neighbor was also found for pure Se, $\mathrm{SSe}_{40}$ and $\mathrm{SSe}_{30}$, respectively, but for $\mathrm{SSe}_{10}$ the bond length increased in comparison with the other compositions. The variations in the coordination number were found to be composition dependent for the prepared samples. The structure was interpreted in terms of the extracted structural parameters and bond angles.
\end{abstract}

\section{Introduction:}

Much work has been done on the thermal, electrical, and optical properties of selenium chalcogenide glasses [1-3]. The selenium sulfur compounds are particularly interesting as they may be easily synthesized in either crystalline or glassy phases. Also these chalcogenide glassy systems are useful for several potential applications such as, threshold switching, memory switching, inorganic photo-resistors, lenses for IR transmission and detection, and optical waveguides, for welding as well as surgery [4-6]. The glass structure of $\mathrm{S}$ and Se have been proposed to be equilibrium mixtures of linear polymer molecules and eight membered monomer rings of concentration $40 \% \mathrm{~S}$ and $60 \%$ Se [7]. Sulfur and Selenium are structurally modeled in the amorphous phase to be either in the random coil or the bundeled coil model [8]. As S and Se are miscible in all proportions [9], the ring-chain structural provides a basis for consideration of the structure of the binary compound SSe. An analysis of Raman spectra for amorphous SSe [10] indicated the formation of fixed eight member rings, possibly $\mathrm{Se}_{5} \mathrm{~S}_{3}$. Although spectroscopic studies have yielded less information on the chain components, theoretical analysis based on equilibrium 
in the liquid phase suggests that the addition of $\mathrm{S}$ reduces the concentration of Se-atoms in the chains as well as the length of the chains [11]. In the present work the structural changes in the amorphous Se caused by the addition of small concentrations of S $(0-9 \%)$ is the aim of the present study.

\subsection{X-ray diffraction experiments and analytical procedure:}

The four compounds, $\mathrm{Se}, \mathrm{SSe}_{40}, \mathrm{SSe}_{30}$ and $\mathrm{SSe}_{10}$ were prepared as bulk glasses by mixing and heating the appropriate constituents of spectroscopically pure elements in an evacuated $\left(1.3 \times 10^{-3} \mathrm{~Pa}\right)$ pyrex ampoules at $623 \mathrm{~K}$ for two hours and subsequently air quenched [12]. Powder X-ray diffraction patterns were recorded, at room temperature, using a Philips diffractometer (X'pert MPD) with Bragg-Brentano parafocusing geometry and graphite monochromated $\mathrm{Cu}-\mathrm{k}_{\alpha}$ radiation. Data were collected over the range $10^{\circ} \leq 2 \theta \leq$ $100^{\circ}$ in $0.02^{\circ} 2 \theta$ steps with an integration time of $3 \mathrm{~s}$, and the collected data were for the as prepared four amorphous samples.

Structure analysis was carried out for the amorphous phases using the RAD program [13].The raw XRD data were corrected for background scattering, counter dead time, polarization and absorption. In the program the missing values between $2 \theta=0^{\circ}$ and $2 \theta=10^{\circ}$ have been found by means of a linear extrapolation. The corrected data were spline smoothed, recalculated in steps of $\Delta \mathrm{K}=1.778 \times 10^{-3} \AA^{-1}$ from $\mathrm{K}=0.0$ to $\mathrm{K}_{\max }=6.24 \AA^{-1}$ (where $\mathrm{K}=4 \pi \sin \theta$ $/ \lambda$; is the scattering vector magnitude ). The data were scaled into electron units by the so-called "high angle method". Only the coherently scattered intensity $\mathrm{I}_{\mathrm{a}}{ }^{\text {coh }}$ was extracted after removing the incoherent (Compton) scattering. The interference functions :

and the reduced RDFs :

$$
I(K)=\left[I_{a}{ }^{c o h}-\sum_{i=1}^{n} x_{i} f_{i}^{2}(K)\right] /\left[\sum_{i=1}^{n} x_{i} f_{i}(K)\right]^{2},
$$

$$
G(r)=2 / \pi \int_{K=0}^{K_{\max }} K I(K) \sin (K r) d K
$$

have been computed according to the Pings and Waser method [14]; where $x_{i}$ is the concentration of the atomic species $i$ having an atomic scattering factor $f_{i}$. Some residual errors in the $\mathrm{I}(\mathrm{K})$, which are mainly occurring as a result of the incorrect normalization of the XRD data, introduce large spurious oscillations in the region between the origin and the first real peak in the corresponding $G(r)$. For preventing such errors a correction was performed by means of repeated Fourier transforms in the way proposed by Kaplow, Strong and Averbach [15]. 


\section{Results and Discussion:}

Figure 1 shows the X-ray diffraction patterns for the four amorphous samples $\mathrm{Se}, \mathrm{SSe}_{40}, \mathrm{SSe}_{30}$ and $\mathrm{SSe}_{10}$. A detailed examination of the patterns indicates that all the samples are amorphous with two main diffraction humps located at $15-40^{\circ}$ and $40-70^{\circ} 2 \theta$. This finding means that the basic structural units are similar in all samples although there is a small angular shift among these samples. The diffraction patterns for $\mathrm{SSe}_{40}, \mathrm{SSe}_{30}$ show hump shifts toward high diffraction angles and a reduction in intensity compared to that of pure Se. The shifts indicate an increase in medium range order (MRO) and the reduction in intensities is due to the replacement of Se atoms by the $\mathrm{S}$ atoms (which have lower atomic scattering factor). In the contrary, the diffraction pattern for $\mathrm{SSe}_{10}$ shows hump shifts toward the lower diffraction angles. Moreover it shows a small shoulder hump at $19-22^{\circ} 2 \theta$, which is more or less similar to the shoulder hump for pure Se and this may indicate an increase in the short range order(SRO) [16] for this concentration. This finding can be also confirmed by finding the inter-cluster spacing (d) from the first sharp diffraction peak (FSDP) using the relation [17-19]:

$$
\mathrm{K}_{\mathrm{FSDP}} \times \mathrm{d}_{\mathrm{MRO}}=7.725
$$

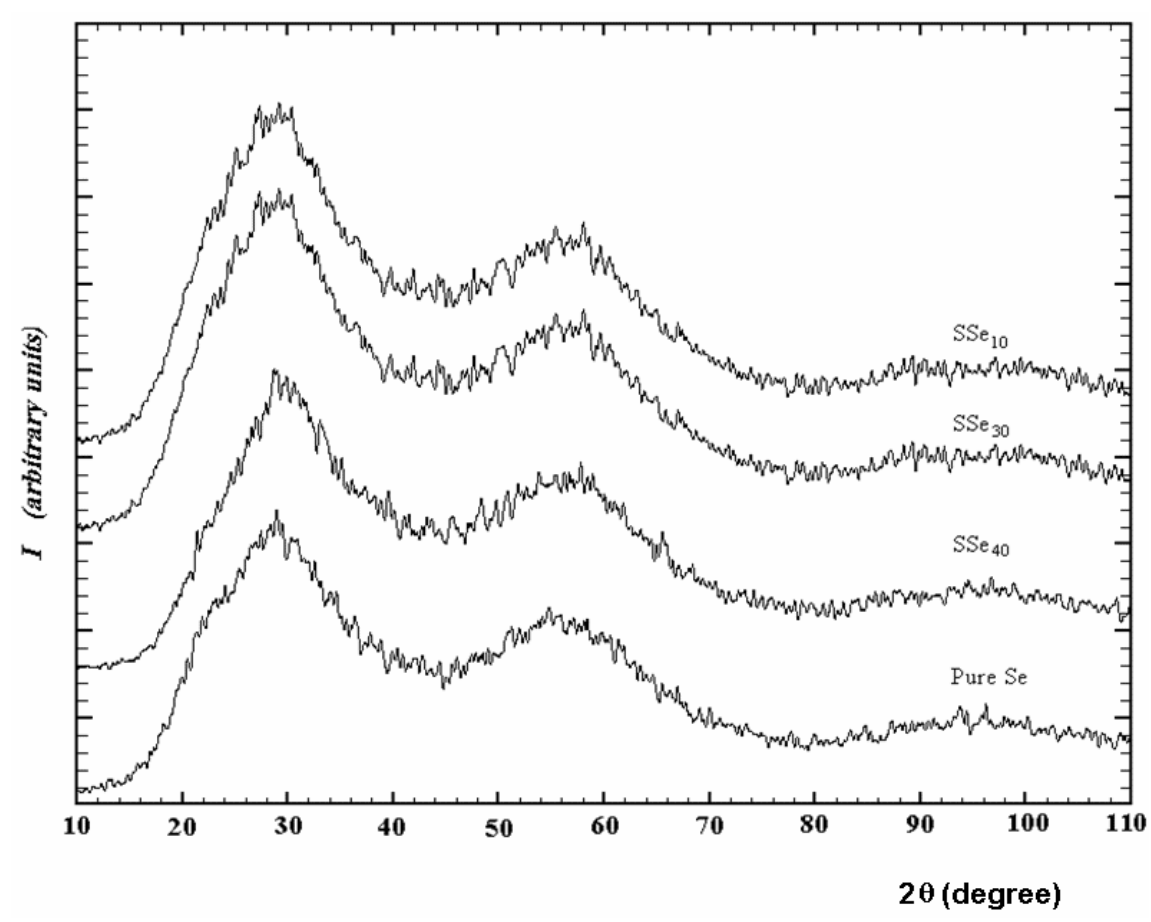

Fig. (1): The scattered intensity (1) versus scattering angle (2 $\theta$ ) for amorphous $\mathrm{Se}, \mathrm{SSe}_{40}, \mathrm{SSe}_{30}$ and $\mathrm{SSe}_{10}$. 
The results of the calculation for the four compositions are given in Table (1).

Table (1): The FSDP in the K-space with the inter-cluster spacing $d$ for the investigated amorphous samples:

\begin{tabular}{|c|c|c|}
\hline Sample & $\mathrm{K}_{\mathrm{FSDP}}\left(\AA^{-1}\right)$ & $\mathrm{d}(\AA)$ \\
\hline $\mathrm{Se}$ & 1.901 & 4.064 \\
\hline $\mathrm{SSe} 40$ & 1.962 & 3.937 \\
\hline $\mathrm{SSe} 30$ & 1.991 & 3.879 \\
\hline $\mathrm{SSe} 10$ & 1.897 & 4.072 \\
\hline
\end{tabular}

It is very clear from the table that the distance between the clusters (of similar motive) is shortest in $\mathrm{SSe}_{30}$ and largest in $\mathrm{SSe}_{10}$. This means that the MRO is highly revealed in $\mathrm{SSe}_{40}$ and $\mathrm{SSe}_{30}$, and by increasing the percentage of $\mathrm{S}$ up to $\sim 9 \%\left(\mathrm{SSe}_{10}\right)$, the $\mathrm{SRO}$ is starting to increase again. The tabulated values 3.937 , and $3.879 \AA$ is more or less closer to the bond length of Se-Se found between two adjacent chains of crystalline structure, (3.46 and $3.47 \AA$ respectively for $\mathrm{SSe}_{40}$ and $\mathrm{SSe}_{30}$ samples) [12]. This finding means that the structural motive of the amorphous materials under study is similar to that of the crystalline materials. The MRO of $\mathrm{SSe}_{40}$ and $\mathrm{SSe}_{30}$ were taking into account the difference between the perfect long range order in case of the crystalline materials and the MRO in the present case of this study. The MRO was also confirmed by measuring the activation energy of crystallization of the four samples considered; where the crystallization energy decreased from 23.36 $\mathrm{Kcal} / \mathrm{mole}$ for Se to $16.17 \mathrm{Kcal} / \mathrm{mole}$ for $\mathrm{SSe}_{30}$, then increased again up to 19.98 $\mathrm{Kcal} / \mathrm{mole}$ for $\mathrm{SSe}_{20}$ [3]. Fig.(2) shows the interference function against $\mathrm{K}$ for the four samples. As can be seen the resolution of the maxima in K-space is good despite the limited K-range in the reciprocal space $(\lambda=1.5405 \AA)$. Fig.(3) gives $\mathrm{RDF}$ versus $\mathrm{R}$ in real space, Table 2 reports the bond length(r), coordination number $(\mathrm{N})$, and the disordering parameter $(\sigma)$ for the observed and simulated RDF results for the first two coordination shells applying the Gaussian shaped model for the simulated shells. Table 3 also reports the estimated bond angles for the given amorphous four samples.

By careful examination of Fig.(3) together with its simulated values as presented in Tables 2 and 3 and as shown in Fig.(4) (for $\mathrm{SSe}_{30}$ sample as an example), one can find the following:

(i) The Se-Se pair of the first coordination shell is observed at $2.358 \AA$ for the smallest and $2.369 \AA$ for the largest values of $\mathrm{S} \%$ among the four samples. That is to say; the deviation in the bond length among the four samples is only $1.1 \times 10^{-3} \AA$; which means that the Se-Se bond of the first nearest neighbor is not largely affected by the addition of $\mathrm{S}$ because it is highly ordered within this limit. From this finding one can conclude that, small 
amount of $\mathrm{S}$ will substitute $\mathrm{Se}$-atoms and since the atomic size of S-atom is smaller $(1.03 \AA)$ than Se-atom $(1.15 \AA)$, then, the bond length decreases between the surrounding Se-atoms, and more arrangement of atoms can take place. While, when the S-atoms increase up to certain limit as in $\mathrm{SSe}_{10}$, some of the $\mathrm{S}$-atoms replace some of Se-atoms, and the rest of $\mathrm{S}$ will be embeded in the Se-Se matrix, and some of the Se-Se bonds will have bridged S-atom, some others will have non-bridged S-atom.

(ii) The coordination number of the first shell is nearly 2.0 with a small decrease in the increased $\mathrm{S} \%$ atoms.

(iii) The Se-Se pairs of the second coordination shell are observed at 3.697, $3.896,3.865$, and $3.853 \AA$ for the four samples and the coordination number of this shell is nearly 4.0 .

(iv) The angles between the bonds within the chain $\left(\varphi_{1}\right)$ for the four amorphous samples are between $103.24^{\circ}$ and $111.41^{\circ}$, but the angles between the bond of the connected atoms among the chains $\left(\varphi_{2}\right)$ are between $77.72^{\circ}$ and $79.84^{\circ}$. The correlation between the experimental and simulated values of both $\varphi_{1}$ and $\varphi_{2}$ is really good. Fig.(5) shows the correlation of $S \%$ for the studied samples against the bond length and bond angles (observed and calculated results). By comparing the above results obtained from the amorphous materials considered with those values obtained from the polycrystalline materials [12], and Fig.(5), one can conclude that: the micro-structural model can explain the structure of the four studied amorphous $\mathrm{SSe}_{\mathrm{x}}$ samples in the $\mathrm{S} \%$ range given.

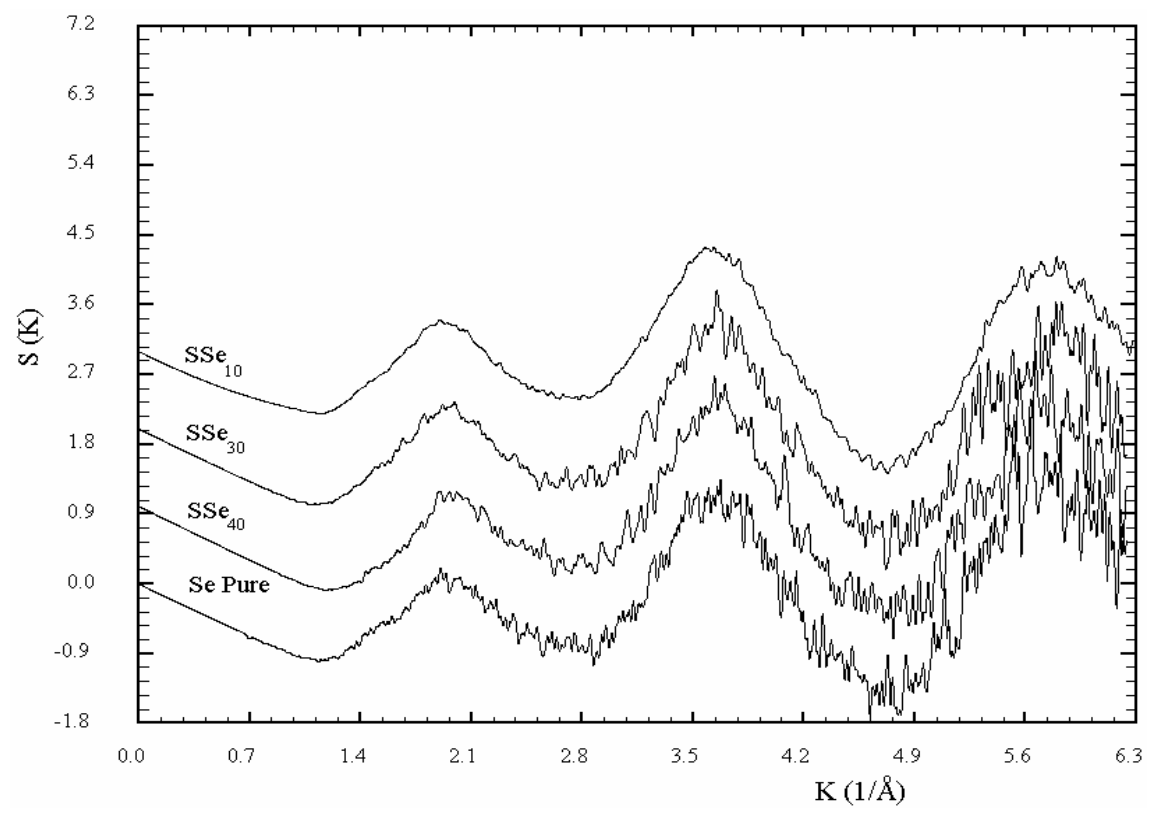

Fig. (2): The interference function of amorphous $\mathrm{Se}, \mathrm{SSe}_{40}, \mathrm{SSe}_{30}$, and $\mathrm{SSe}_{10}$. 


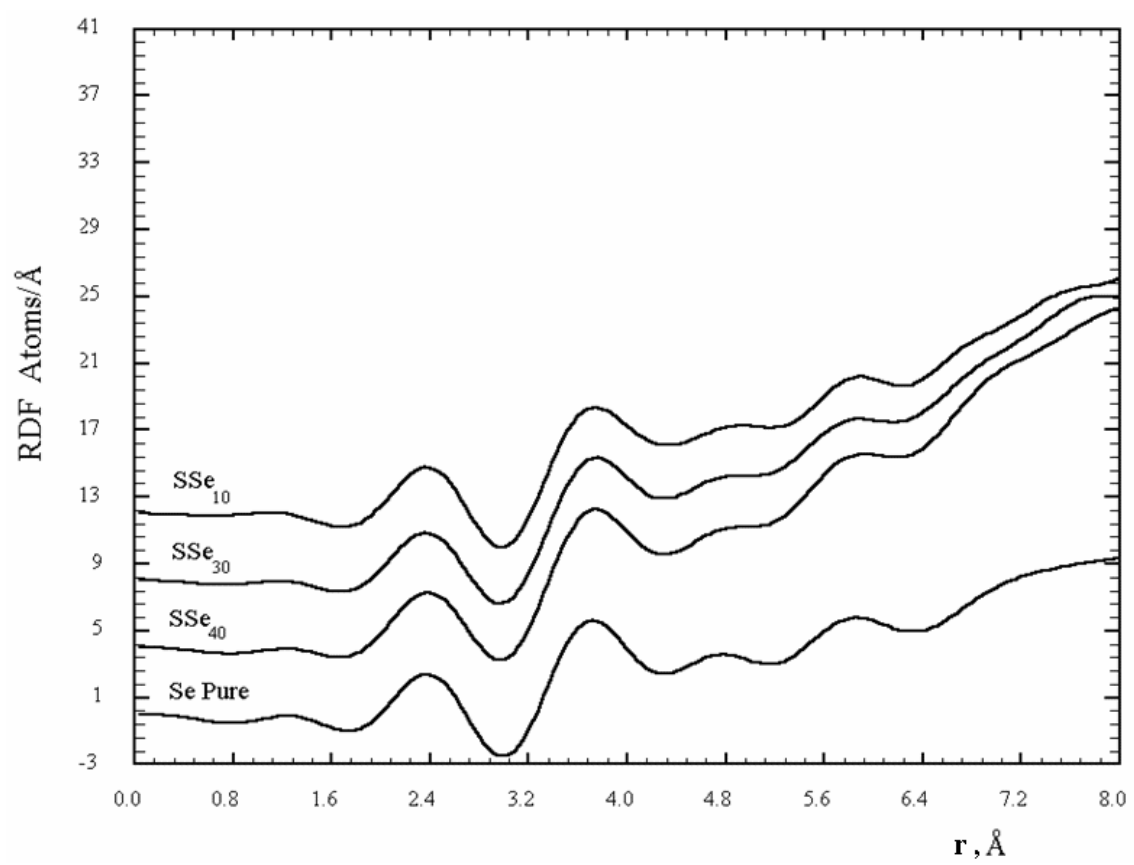

Fig. (3): RDF against $\mathrm{R}$ of amorphous $\mathrm{Se}, \mathrm{SSe}_{40}, \mathrm{SSe}_{30}$ and $\mathrm{SSe}_{10}$ samples.

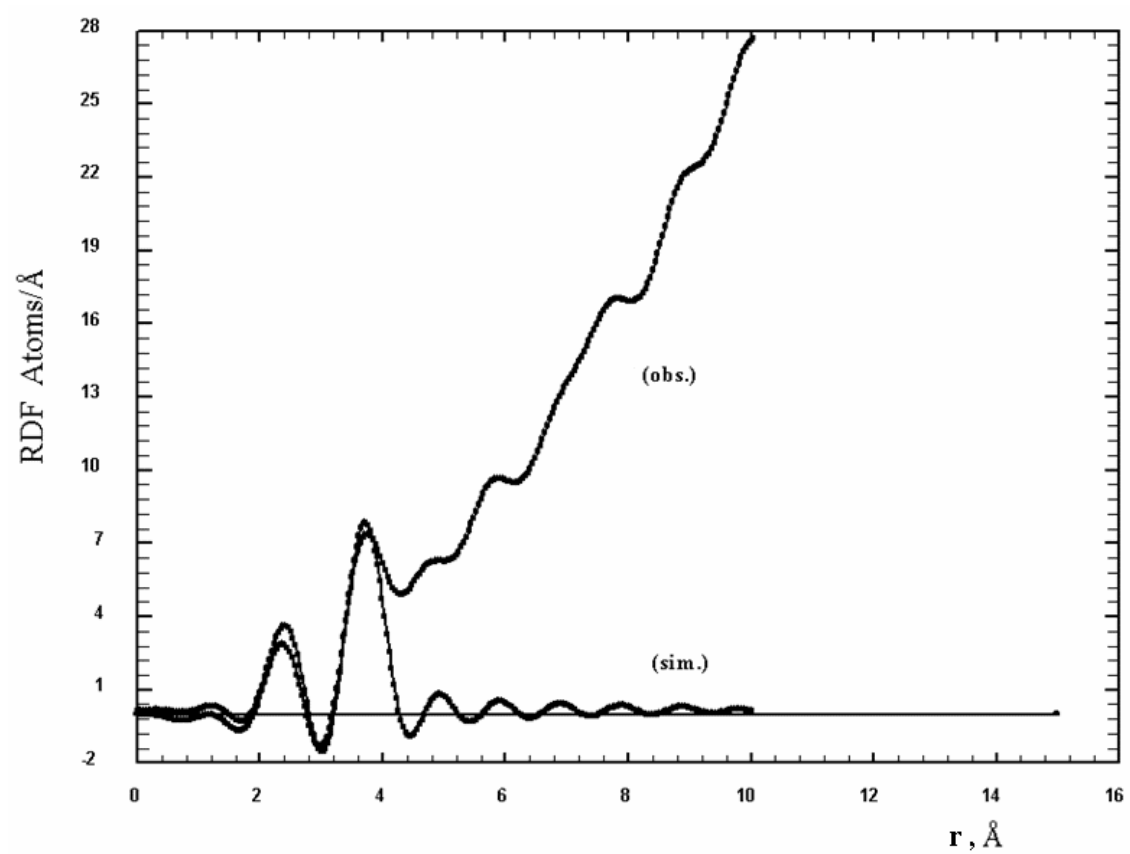

Fig. (4): The simulation of the first two coordination shells for amorphous $\mathrm{SSe}_{30}$. 


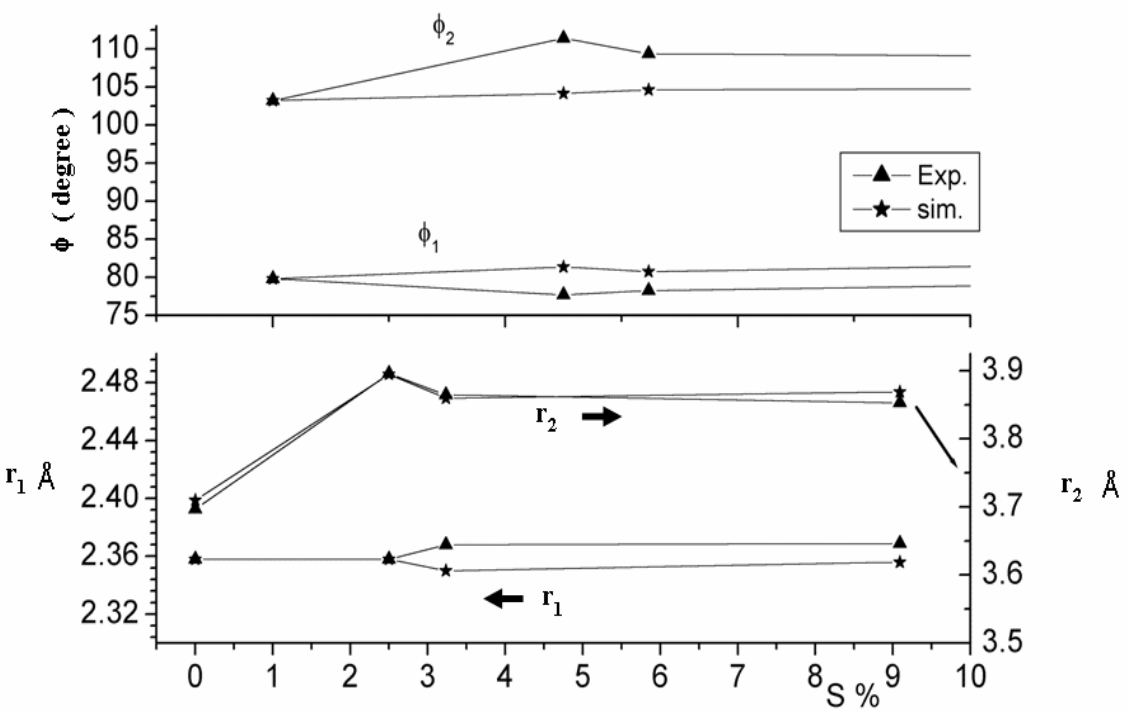

Fig. (5): The correlation of $S \%$ with the bond lengths $r_{1}$ and $r_{2}$, and with the bond angles $\varphi_{1}$ and $\varphi_{2}$ for the studied amorphous samples.

Table.(2): Observed and Simulated RDF results of the studied samples:

\begin{tabular}{|c|c|c|c|c|c|c|}
\hline Sample & $\mathrm{r}_{1}(\AA) \mathrm{obs}$ & $\mathrm{r}_{1}(\AA) \operatorname{sim}$ & $\mathrm{N}_{1}$ & $\overline{N_{1}(\operatorname{sim})}$ & $\sigma_{1}(\AA)$ obs & $\sigma_{1}(\AA) \operatorname{sim}$ \\
\hline $\mathrm{a}-\mathrm{Se}$ & 2.358 & 2.358 & 2.200 & 1.550 & 0.630 & 0.0099 \\
\hline a-SSe 40 & 2.358 & 2.358 & 2.160 & 1.580 & 0.650 & 0.070 \\
\hline $\mathrm{a}-\mathrm{SSe}_{30}$ & 2.368 & 2.350 & 2.140 & 1.550 & 0.635 & 0.065 \\
\hline$\overline{\mathrm{a}-\mathrm{SSe}_{10}}$ & 2.369 & 2.356 & 2.096 & 1.500 & 0.640 & 0.016 \\
\hline
\end{tabular}

Table.2 Contin.

\begin{tabular}{|c|c|c|c|c|c|c|}
\hline Sample & $\mathrm{r}_{2}(\AA) \mathrm{obs}$ & $\mathrm{r}_{2}(\AA) \operatorname{sim}$ & $\mathrm{N}_{2}$ & $\mathrm{~N}_{2}(\operatorname{sim})$. & $\sigma_{2}(\AA)$ obs & $\sigma_{2}(\AA) \operatorname{sim}$ \\
\hline $\mathrm{a}-\mathrm{Se}$ & 3.697 & 3.710 & 4.250 & 3.25 & 0.900 & 0.0999 \\
\hline $\mathrm{a}-\mathrm{SSe}_{40}$ & 3.896 & 3.895 & 4.644 & 4.90 & 0.950 & 0.150 \\
\hline $\mathrm{a}-\mathrm{SSe}_{30}$ & 3.865 & 3.860 & 4.192 & 4.45 & 0.930 & 0.150 \\
\hline $\mathrm{a}-\mathrm{SSe}_{10}$ & 3.853 & 3.869 & 4.166 & 4.20 & 0.936 & 0.160 \\
\hline
\end{tabular}

$\sigma_{t}=\sqrt{\sigma^{2}+\sigma^{2}{ }_{\text {sim }}}$

$\sigma_{\mathrm{t}}$ is the total disordered parameter, both of $\sigma_{1}$ and $\sigma_{1}(\operatorname{sim}$.$) in \AA$ represents the observed(or thermal) and simulated disordering parameters. 
Table (3): Estimated bond angles for the given amorphous four samples:

\begin{tabular}{|c|c|c|c|c|}
\hline Sample & $\Phi_{1}{ }^{\text {a }}$ (obs. $)$ & $\Phi_{1}{ }^{\circ}(\operatorname{sim})$. & $\Phi_{2}{ }^{\circ}$ (obs. $)$ & $\Phi_{2}{ }^{\circ}(\operatorname{sim})$. \\
\hline $\mathrm{a}-S e$ & 103.24 & 103.24 & 79.84 & 79.84 \\
\hline a-SSe & 11.41 & 104.15 & 77.72 & 81.37 \\
\hline a-SSe & 109.39 & 104.65 & 78.27 & 80.76 \\
\hline a-SSe & 108.82 & 104.87 & 79.55 & 82.19 \\
\hline
\end{tabular}

${ }^{\text {a }}$ The angle in a chain, presumably between the two primary bonds.

\section{Conclusion:}

The micro-structural model can explain in a significant way the structure of the amorphous $\mathrm{SSe}_{\mathrm{x}}$ samples in the $(\mathrm{x}=10-40) \mathrm{S} \%$ range given. Also, in pure Se and the sample $\mathrm{SSe}_{10}$, the $\mathrm{SRO}$ is highly declared and the added S-atoms in the matrix will be interstitially arranged. In contradictory, the two samples $\mathrm{SSe}_{30}$ and $\mathrm{SSe}_{40}$ have a MRO of large declaration and the added $\mathrm{S}$-atoms in the matrix are substituting the Se-atoms; causing a shortening in the Se-Se bond lengths and a decreasing in their coordination numbers. The obtained results are confirmed by both the study of crystallization kinetics and bond angles distribution for the given samples.

\section{Acknowledgements:}

I would like to acknowledge sincerely Prof. Dr. M.B. El Den for providing the amorphous samples $\left(\mathrm{a}-\mathrm{SSe}_{\mathrm{x}}\right)$. Also, I am indebted to Prof. Dr. Z.K. Heiba for his valuable discussions. A lot of thanks to Prof. Dr. K.El-Sayed for the revision of the manuscript and for efficient fruitful discussions and continous encouragement.

\section{References:}

1. M.K. El-Mously, M.F. Kotkata and M.A. Salem, J.Phys.C11, 1077 (1978).

2. Jecu-Donia, Jaklovsky-Jeno, Trutia Atanasie, Apostol Ileana, Dinescu Maria, Michailescu Ion N, Aldica Gheorghe, Popescu Mihai, Vlahovici Nicolae, Zamfira Sorin and Indrea Emil, J. Non-Crystalline Solids 90, 1-3 (1986).

3. L.A. Wahab, M.B. El Den and M.F. Youssof, Egypt. J. Solids 23, 71 (2000).

4. S.R. Ovshinsky, Phys. Rev. Letters 21, 1450 (1988).

5. L.A. Wahab, "Proceeding of the International Conference (Science \& development \& Environment)", Cairo, 215 (1997). 
6. A.F. Maged, L.A. Wahab and I.A. El Kholy, J. Materials Science 33, 3331 (1998).

7. J. Schottmiller, M. Tabak, G. Lucovsky and A. Ward, J. Non-Crystalline Solids 4, 80 (1970).

8. A.C. Wright, "Proceedings of the Egyptian $4^{\text {th }}$ International School of Crystallography", Edited by K. El-Sayed and M. Radwan, Aswan, 564 (1993).

9. I.A. Aleksandrovich, Uch. Zap. Leningrad Gas Pedagog. Inst. 202, 231 (1966).

10. N.M. Mott and E.A. Davis, "Electronic Processes in Non-Crystalline Materials", Clarendson Oxford, (1971).

11. M.B. Myers and E.J. Felty, Mater. Res. Bull. 2, 535 (1967).

12. Z.K. Heiba, M.B. El-Den and Karimat El-Sayed, Powder Diffraction 17(2), 1 (2002).

13. V. Petkov, A. Apostolov and V. Skumryev, J. Non-Crystalline Solids 108, 75 (1989).

14. C. Pings and J. Waser, J. Chem. Phy. 48, 3016 (1968).

15. R. Kaplow, S. Strong and B. Averbach, Phys. Rev. A138, 1336 (1965).

16. A. Abou Zeid Mohamed, "The Structure of Chalcogenide Glasses in the Ge-Sb-S System", Ph. D. Thesis, Assiut University (1990).

17. L. Cervinka, J. Komrska and J. Mikes, J. Non-Crystalline Solids 75, 69 (1985).

18. L. Cervinka, J. Non-Crystalline Solids 97, 98, 207 (1987).

19. L. Cervinka, J. Non-Crystalline Solids 90, 371 (1987). 